\title{
NEONATAL MORTALITY IN SOUTH-WEST OF IRAN: RATES AND ASSOCIATED FACTORS
}

\author{
N. Maharlouei ${ }^{1}$, L. Maani ${ }^{2}$, K.B. Lankarani ${ }^{1}$ \\ ${ }^{1}$ Health Policy Research Center, ${ }^{2}$ Vice-Chancellor for Clinical Affairs, Shiraz University of Medical \\ Sciences, Shiraz, Iran
}

Object: To determine neonatal mortality rate, and its associated factors we conduct this study as a basis for the following surveys.

Method: From March21, 2009 to March20, 2010 all neonatal mortality occurred in Fars province, the fifth populated province in Iran, were considered in this cross sectional study. Data had been recorded in details containing demographic part (maternal and neonatal), obstetric history, and medical records.

Result: Recorded neonatal mortality rate in this period was 1270.6 per100000 living birth. 555 out of 864 $(67.4 \%)$ occurred within the 24 hours after birth and 737 (89.5\%) during the first week. 573 out of 864 (77.4\%) weighted less than 2500 grams and 422 (48.8\%) neonates were less than 1500 grams. The most recorded reasons of neonatal mortality (23\%) were related to prematurity, while 147 out of $864(17 \%)$ had congenital anomaly. Most of mothers (96.1\%) gave birth in hospital and $99.7 \%$ were under supervision of a gynecologist or a midwife ( $(70.6 \%)$ ). Mean age of mothers were $27.66 \pm 6.17$ years and $13.8 \%$ had an underlying disease, however $2.3 \%$ reported medicine consumption, mostly aspirin $(20.8 \%)$ and antibiotics $(16.6 \%)$.

Conclusion: Despite good access to professional care, we found a high neonatal mortality rate, often due to preventable conditions. These results suggest that to decrease neonatal mortality rate, preventing prematurity is essential. 